\title{
Regulatory network analysis of genes and microRNAs in human hepatoblastoma
}

\author{
JIMIN HE ${ }^{1,3}$, XIAOXIN GUO ${ }^{1-3}$, LINLIN SUN ${ }^{2,3}$, NING WANG ${ }^{1,3}$ and JIWEI BAO ${ }^{2,3}$ \\ ${ }^{1}$ College of Computer Science and Technology; ${ }^{2}$ College of Software; ${ }^{3}$ Key Laboratory of Symbolic Computation \\ and Knowledge Engineering of Ministry of Education, Jilin University, Changchun, Jilin 130012, P.R. China
}

Received March 12, 2015; Accepted January 11, 2016

DOI: $10.3892 / \mathrm{ol} .2016 .5196$

\begin{abstract}
Hepatoblastoma (HB) is a common type of primary tumor in children. Previous studies have examined the expression of genes, including transcription factors (TFs), target genes, host genes and microRNAs (miRNAs or miRs) associated with HB. However, the regulatory pathways of miRNAs and genes remain unclear. In the present study, a novel perspective is proposed, which focuses on HB and the associated regulatory pathways, to construct three networks at various levels, including a differentially expressed network, an associated network and a global network. Genes and miRNAs are considered as key factors in the network. In the three networks, the associations between each pair of factors, including TFs that regulate miRNAs, miRNAs that interact with target genes and miRNAs that are located at host genes, were analyzed. The differentially expressed network is considered to be the most crucial of the three networks. All factors in the differentially expressed network were mutated or differentially expressed, which indicated that the majority of the factors were cancerogenic factors that may lead to HB. In addition, the network contained numerous abnormal linkages that may trigger HB. If the expression of each factor was corrected to a normal level, HB may be successfully treated. The associated network included more HB-associated genes and miRNAs, and was useful for analyzing the pathogenesis of HB. By analyzing these close associations, the first and the last factor of the regulatory pathways were revealed to have important roles in HB. For example, v-myc avian myelocytomatosis viral oncogene neuroblastoma derived homolog (MYCN) was observed to regulate
\end{abstract}

Correspondence to: Professor Xiaoxin Guo, College of Computer Science and Technology, Jilin University, 2,699 Qianjin Street, Changchun, Jilin 130012, P.R. China

E-mail: guoxx@jlu.edu.cn

Abbreviations: HB, hepatoblastoma; miRNA, microRNA; TF, transcription factor; TFBs, transcription factors binding sites; NCBI, National Center for Biotechnology Information; FFL, feed-forward loop

Key words: hepatoblastoma, transcription factors, host gene, microRNA, target genes, network
Homo sapiens (hsa)-miR-221, hsa-miR-18a and hsa-miR-17-5p, but no miRNAs targeted MYCN. In conclusion, the pathways and mechanisms underlying $\mathrm{HB}$ were expounded in the present study, which proposed a fundamental hypothesis for additional studies.

\section{Introduction}

Hepatoblastoma (HB) is the most common hepatic malignancy in children, and accounts for $\sim 1 \%$ of all childhood tumors (1). Usually, the disease is diagnosed during the first three years of a child's life (2). The etiology of HB is unclear, but has been associated with Beckwith-Wiedemann syndrome, familial adenomatosis polypi and low birth weight (3). The primary treatment for HB is surgical resection, and the use of pre-resection chemotherapy may increase the likelihood of a resectable tumor (3). Therefore, the pathogenesis of HB requires urgent understanding.

Differentially expressed genes and microRNAs (miRNAs or miRs) have been previously indicated to be crucial in HB, affecting the development, metastasis and treatment of this disease (4). HB-associated factors have less effect, compared with differentially expressed factors (5). Despite the large number of genes and miRNAs that have been reported to affect $\mathrm{HB}$, the association between them remains unclear.

Transcription factors (TFs), miRNAs and target genes construct an intricate regulatory network that provides a novel opportunity to study HB (6). TFs and miRNAs are prominent regulators of gene expression. Gene regulatory factors are predominantly comprised of TFs and miRNAs, which control the expression of genomic information in multicellular genomes (7). TFs are proteins that bind to specific DNA sequences, thus controlling the transfer of genetic information between DNA and messenger (m)RNA (8). In the three networks at various levels described in the present study, TFs regulate miRNAs and miRNAs target other genes. As a result, TFs influence the expression of genes via miRNAs. miRNAs are endogenous RNAs of $\sim 23 \mathrm{nt}$ in length that are crucial for gene regulation, as they interact with the mRNAs of protein-coding genes to control their post-transcriptional repression (9). Magrelli et al (10) demonstrated that miRNAs associated with carcinogenesis may play a pivotal role in the onset of HB. TFs and miRNAs interact with each other (11). Wang et al (12) reported that TFs regulate the expression of miRNAs, and miRNAs regulate the 
transcription of TFs. This complex association is included in the networks of the present study.

Target genes are targeted by miRNAs (13). Naeem et al (14) indicated that miRNAs may exert a widespread impact on the regulation of target and non-target genes. At present, miRNAs are known to target numerous genes (15). These target genes, for which abundant information may be obtained from available databases and pertinent literature (16), may provide essential evidence for the discovery of the biological role of miRNAs (17).

Host genes are genes where miRNAs are located. Rodriguez et al (18) suggested that the transcriptional patterns of all miRNA host genes were derived from a variety of sources that illustrate the spatial, temporal and physiological regulation of miRNA expression. This theory indicates that miRNAs are transcribed in parallel with their host transcripts, and that the two different transcription classes of miRNAs identified ('exonic' and 'intronic') may require slightly different mechanisms of biogenesis (18). The cooperation of miRNAs with the host gene may affect the disease process (19). In the present study, the host genes are considered to be mutated and involved in the progression of cancer when their corresponding miRNA is differentially expressed.

From the experimental data obtained in the present study, the differentially expressed genes and miRNAs were concluded to have a paramount impact on HB. In addition, the associated genes and miRNAs also exert certain effect on HB.

In the present study, the underlying networks of HB were assessed with respect to miRNAs, target genes, TFs, host genes of miRNAs and the associations between these factors in human HB. Various data were collected, and the associations between the aforementioned factors were revealed to be intricate. The differences and similarities between these factors were compared, and significant associations were extracted for analysis, in order to aid the understanding of the pathogenesis of $\mathrm{HB}$.

\section{Materials and methods}

\section{Material collection and data processing}

Extraction of the regulatory associations between miRNAs and target genes. The interactions between human miRNAs and target genes were extracted from two databases, TarBase 5.0 (20) and miRTarBase (http://mirtarbase.mbc.nctu.edu.tw). The gene symbols used in the present study were adapted from official symbols in the National Center for Biotechnology Information (NCBI) database (http://www.ncbi.nlm.nih.gov/gene). The miRNA symbols were normalized using miRBase (http://www.mirbase.org). All the information extracted was termed dataset D1.

Extraction of the regulatory associations between TFs and miRNAs. The experimentally validated dataset of human TFs and the miRNAs regulated by these TFs was collected from TransmiR (12), a manually extracted database that details the associations between TFs and miRNAs. The TF and miRNA association data were collected into the dataset D2.

Extraction of the regulatory associations between miRNAs and host genes. The host genes of human miRNAs were extracted from the miRBase (21) and NCBI databases. In order to ensure the accuracy of the present study, each host gene was manually assigned an official symbol and an official identity. The miRNA and host gene data were included in the dataset D3.

Extraction of data regarding genes and miRNAs. The differentially expressed genes in HB were collected from the Cancer GeneticsWeb (www.cancerindex.org/geneweb), the NCBI database of single nucleotide polymorphism (dbSNP) (www.ncbi.nlm.nih.gov/snp) and pertinent literature in the Science Citation Index (22). The differentially expressed genes were termed dataset D4. The associated genes were then extracted from GeneCards ${ }^{\circledR}$ (www.genecards.org) and pertinent literature.

Predicted TFs were identified from target genes of the differentially expressed miRNAs. The TFs were extracted using the P-Match method (http://www.gene-regulation.com/ pub/programs.html\#pmatch), an algorithm that operates on the concepts of pattern matching and weight matrix approaches in order to identify transcription factor binding sites (TFBSs) in DNA sequences (23). The genes that appeared in TransmiR were regarded to be $\mathrm{HB}$-associated genes, and were further analyzed. In total, 1,000 nt promoter region sequences of the targets of differentially expressed genes were downloaded from the University of California Santa Cruz database (24). P-Match was used to combine pattern matching and weight matrix approaches in order to identify the TFBSs in $1,000 \mathrm{nt}$ promoter region sequences. The TFBSs were mapped onto the promoter regions of the targets. The P-Match matrix library consists of a set of known TFBSs that were collected from the TRANSFAC ${ }^{\circledR}$ database (http://www.gene-regulation.com/pub/databases.html), thus providing the possibility to search for large numbers of differentially expressed TFBSs. The differentially expressed genes that were obtained were also associated genes. The data regarding differentially expressed genes and associated genes were termed dataset D5.

The differentially expressed miRNAs were extracted from pertinent literature and miR2Disease $(25,26)$, which is a manually curated database regarding differentially expressed miRNAs in HB. The differentially expressed miRNAs were termed dataset D6. The associated miRNAs were collected from pertinent literature. The differentially expressed miRNAs were also associated miRNAs. The associated miRNAs collected were termed dataset D7.

Construction of three networks at various levels. The differentially expressed network, associated network and global network were constructed. All the regulatory associations depended upon host genes, target genes, miRNAs and TFs. Specifically, the associations between host genes, miRNAs, target genes and TFs were extracted. Through combining all the associations in datasets D1-D7, the global network was obtained. The differentially expressed network and associated network were obtained separately from the global network, and the associated network contained the differentially expressed network.

The differentially expressed network was identified as the most crucial network, since genes and miRNAs are the primary factors to construct this complex network. The factors and pathways were obtained by mapping the differentially expressed genes and miRNAs datasets D4 and D6 onto datasets D1, D2 and D3. Then, the regulatory pathways were used to construct the network. 


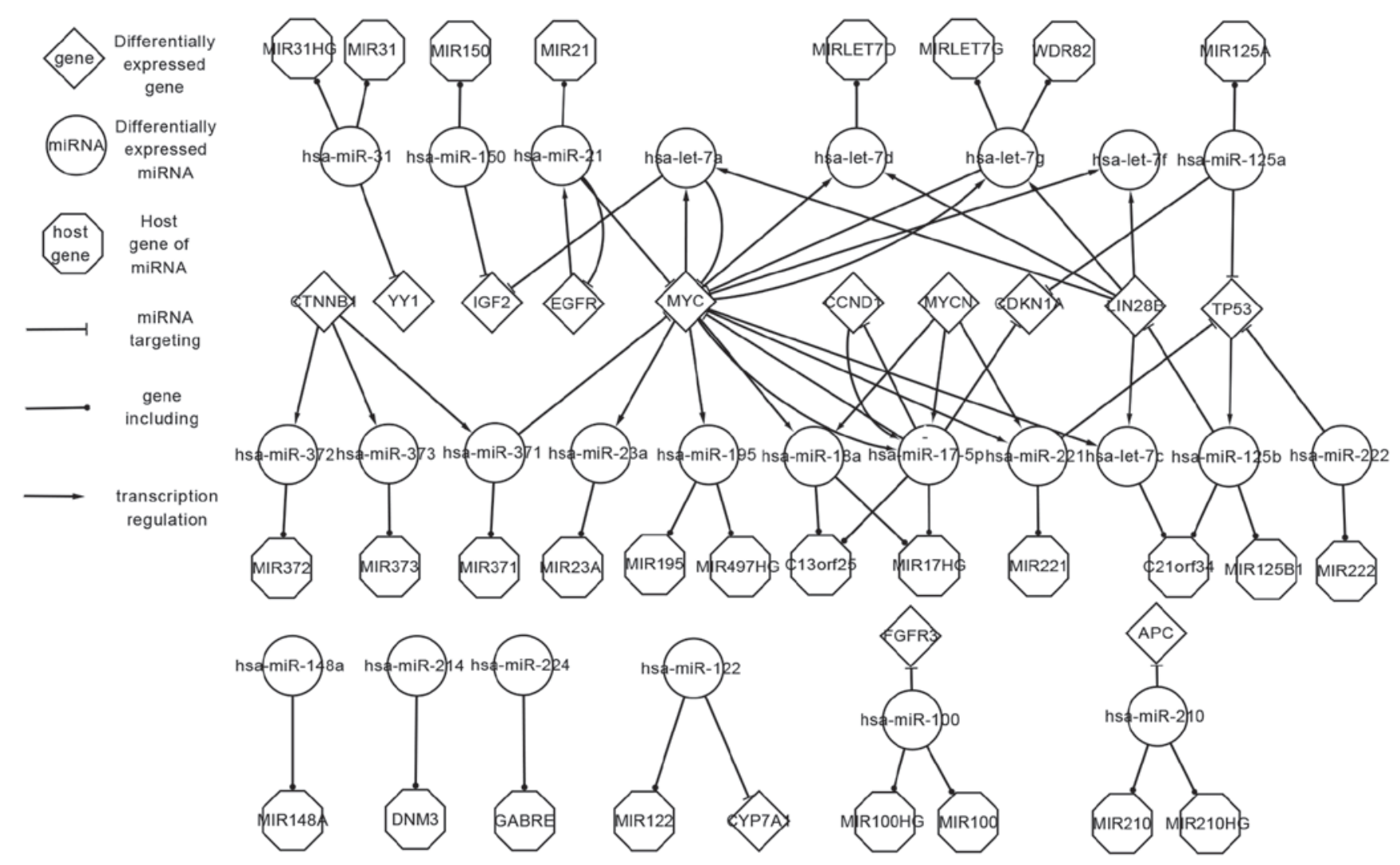

Figure 1. Differentially expressed network of genes and microRNAs that are differentially expressed in hepatoblastoma. miRNA; microRNA; MIR, microRNA; miR, microRNA; HG, host gene; hsa, Homo sapiens; let, lethal; WDR82, WD repeat-containing protein 82; CTNNB1, catenin beta 1; YY1, Yin Yang 1; IGF2, insulin-like growth factor 2; EGFR, epidermal growth factor receptor; MYC; v-myc avian myelocytomatosis viral oncogene homolog; MYCN, v-myc avian myelocytomatosis viral oncogene neuroblastoma derived homolog; CCND1, cyclin D1; CDKN1A, cyclin-dependent kinase inhibitor 1A; LIN28B, lin-28 homolog B; TP53, tumor protein p53; C13orf25, MIR17HG; C21orf34, MIR99AHG; FGFR3, fibroblast growth factor receptor 3; APC, adenomatous polyposis coli; DNM3, dynamin 3; GABRE, gamma-aminobutyric acid receptor subunit epsilon; CYP7A1, cytochrome P450 family 7 subfamily A member 1.

Differentially expressed genes, associated genes and the respective miRNAs are all involved in the process of HB. As the associated genes and miRNAs are also involved in the process of $\mathrm{HB}$, the datasets D5 and D7 were mapped onto the datasets D1, D2 and D3. The associated network, including the differentially expressed network and other factors, demonstrated intricate associations.

The differentially expressed and associated networks are hypothesized to be important for investigating HB. However, with the exception of the experimentally validated genes and miRNAs present in these two networks, certain data that have no direct association with HB may be involved in the process of HB. All the TFs and miRNAs that were present in the associated network were mapped onto the datasets D1, D2 and D3 to obtain the global network.

\section{Results}

Differentially expressed network of $H B$. A large number of notable regulatory associations and factors are shown in Fig. 1. The network consists of 6 TFs [namely catenin beta 1 (CTNNB1), epidermal growth factor receptor (EGFR), v-myc avian myelocytomatosis viral oncogene homolog (MYC), v-myc avian myelocytomatosis viral oncogene neuroblastoma derived homolog (MYCN), lin-28 homolog B (LIN28B) and cyclin D1 (CCND1)], 25 miRNAs, 9 target genes and 30 host genes.
The complex associations between genes and miRNAs may be classified into three classes: i) TFs that regulate miRNAs; ii) miRNAs that locate at host genes; iii) and miRNAs that interact with target genes. The reasons behind the intricate associations in the differentially expressed network may be summarized as follows: Firstly, a single miRNA may be located at $>1$ host gene, and several miRNAs may be located at the same host genes (27). For example, Homo sapiens (hsa)-miR-210 is located at the genes MIR210 and MIR210 host gene (HG), while hsa-miR-18a and hsa-miR-17-5p are located at MIR17HG (also known as C13orf25). Secondly, certain TFs not only regulate miRNAs, but also act as target genes. For example, LIN28B regulates hsa-lethal (let)-7a, hsa-let-7c, hsa-let-7d, hsa-let-7g and hsa-let-7f, and is also targeted by hsa-miR-125b. In addition, certain feed-forward loops (FFLs) are important to consider. For example, MYC and CCND1 regulate hsa-miR-17-5p, which in turn targets MYC and CCND1.

The upstream and downstream information may describe the association between genes and miRNAs more clearly. For example, LIN28B and MYC regulate hsa-let-7a, which in turn targets insulin like growth factor 2 (IGF2) and MYC. Therefore, the upstream genes LIN28B and MYC impact the downstream genes IGF2 and MYC through hsa-let-7a.

The differentially expressed network provides important information regarding the regulatory mechanisms of HB. 




Figure 2. Transcriptional network of transcription factors and microRNAs involved in hepatoblastoma..MIR, microRNA; HG, host gene; C21orf34, MIR99AHG; hsa, Homo sapiens; let, lethal; EGFR, epidermal growth factor receptor; CCND1, cyclin D1; MYC, v-myc avian myelocytomatosis viral oncogene homolog; MYCN, v-myc avian myelocytomatosis viral oncogene neuroblastoma derived homolog; CTNNB1, catenin beta 1; LIN28B, lin-28 homolog B; TP53, tumor protein p53; C13orf25, MIR17HG; WDR82, WD repeat-containing protein 82.

Transcriptional network of $H B$. The TFs that were included in the differentially expressed network were further analyzed. Fig. 2 shows the regulatory pathways of TFs, differentially expressed miRNAs and host genes that associate with each other, according to the differentially expressed network. These factors affect their downstream elements by regulating or targeting them.

In total, 7 TFs, namely EGFR, CCND1, MYCN, MYC, CTNNB1, LIN28B and tumor protein 53 (TP53) are shown in Fig. 2. MYC and MYCN affect CCND1 through hsa-miR-17-5p, while hsa-miR-21 and EGFR form a FFL.

MYC is a crucial TF in the differentially expressed network, since it regulates hsa-miR-195,-221,-18a and -17-5p, in addition to hsa-let-7a, c, d, f and g. Of these miRNAs, hsa-miR-17-5p targets CCND1, which indicates that MYC affects CCND1 through hsa-miR-17-5p. Cairo et al (28) indicated that hsa-miR-100, hsa-miR-371, hsa-miR-373 and hsa-let-7a, also known as the four-miR signature, may be used to classify $\mathrm{HB}$, and reported that MYC activation is important in the pathogenesis of HB.

Previously, MYCN was indicated to be highly unregulated in HB (4). However, the results of the present study indicate that MYCN regulates hsa-miR-221, hsa-miR-17-5p and hsa-miR-18a. Furthermore, CCND1 and EGFR were previously reported to exhibit differentially increased expression in HB (29).

According to the pathways constructed in a previous study, hsa-let-7a targets MYC, thus exerting tumor suppressive activity (28). A previous study indicated that the targeting of the LIN28B gene by MYC contributes to the regulation of miRNA expression, notably by inhibiting the biogenesis of hsa-let-7a, c, d, f and g (30). In addition, the expression of LIN28B was negative correlated with the expression of the aforementioned miRNAs (31).

The transcriptional network, including genes, miRNAs and their associations, meaningfully explains the pathogenesis of $\mathrm{HB}$.

Associated network of HB. Fig. 3 shows the associated network of associated expressed genes, associated expressed miRNAs and the host genes of associated expressed miRNAs. The associated network includes all the factors and pathways that are included in the differentially expressed network.

With the exception of the factors included in the differentially expressed network, the associated network contained additional 9 TFs, namely CCAAT/enhancer binding protein alpha, hepatic nuclear factor 4 alpha, nuclear factor of kappa light polypeptide gene enhancer in B-cells 1 (NFKB1), v-rel avian reticuloendotheliosis viral oncogene homolog (REL), RELA, CTNNB1, nuclear receptor subfamily 1 , group H, member 4 (NR1H4), zinc finger E-box binding homeobox 1 (ZEB1) and NK2 homeobox 5. Four of these TFs, namely ZEB1, REL, NFKB1 and RELA, were obtained using the P-Match algorithm. With the exception of differentially expressed miRNAs, 12 associated miRNAs, including hsa-miR-122, hsa-miR-492 and hsa-miR-34a, are shown in the associated network (Fig .3). These associated factors affect the therapy, prognosis and clinical research of HB (32).

An attractive miRNA (hsa-miR-34a) is shown in Fig. 3. This miRNA targets 13 genes, including mesenchymal epithelial transition factor (MET), cyclin-dependent kinase (CDK)4 


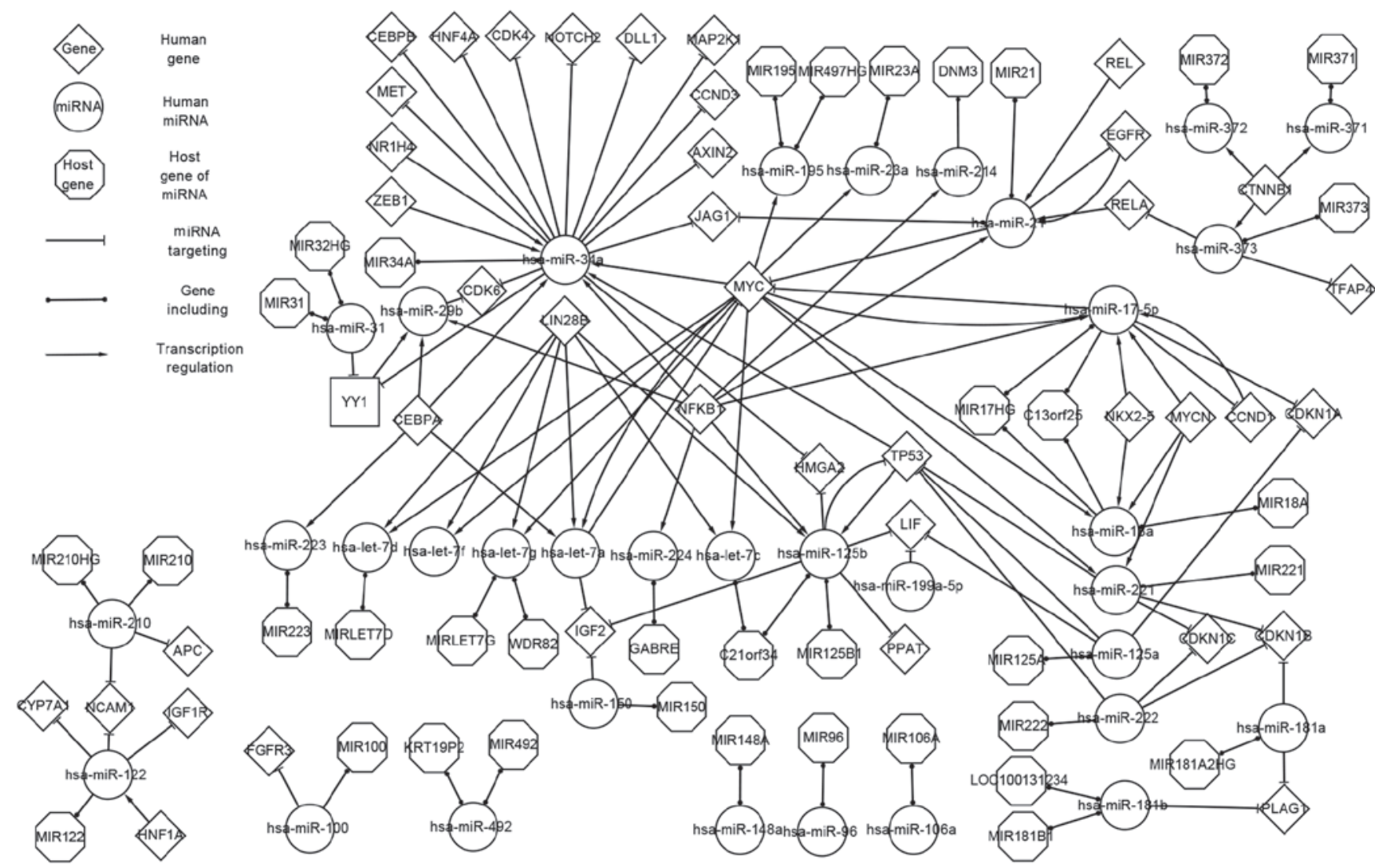

Figure 3. Associated network describing the associations between genes and microRNAs involved in hepatoblastoma. miRNA; microRNA; CEBPB, CCAAT/enhancer-binding protein beta; HNF, hepatocyte nuclear factor; $\mathrm{CDK} 4$, cyclin-dependent kinase 4; NOTCH2, neurogenic locus notch homolog protein 2; DLL1, delta-like 1; MAP2K1, mitogen-activated protein kinase kinase 1; MET, mesenchymal epithelial transition factor; NR1H4, nuclear receptor subfamily 1, group H, member 4; ZEB1, zinc finger E-box binding homeobox 1; CCND3, cyclin D3; AXIN2, axis inhibition protein 2; JAG1, jagged 1; hsa, Homo sapiens; miR, microRNA; MIR, microRNA; HG, host gene; CDK6, cyclin-dependent kinase 6; LIN28B, lin-28 homolog B; YY1, Yin Yang 1; CEBPA, CCAAT/enhancer-binding protein alpha; NFKB1, nuclear factor of kappa light polypeptide gene enhancer in B-cells 1; MYC, v-myc avian myelocytomatosis viral oncogene homolog; MYCN, v-myc avian myelocytomatosis viral oncogene neuroblastoma derived homolog; DNM3, dynamin 3; REL, v-rel avian reticuloendotheliosis viral oncogene homolog; EGFR, epidermal growth factor receptor; CTNNB1, catenin beta 1; TFAP4, transcription factor activating enhancer-binding protein 4; C13orf25, MIR17HG; NKX2-5, NK2 homeobox 5; CCND1, cyclin D1; CDKN1, cyclin-dependent kinase inhibitor 1; LOC100131234, MIR181A1HG; PLAG1, pleomorphic adenoma gene 1; HMGA2, high mobility group AT-hook 2; TP53, tumor protein p53; LIF, leukemia inhibitory factor; PPAT, phosphoribosyl pyrophosphate amidotransferase; C21orf34, MIR99AHG; GABRE, gamma-aminobutyric acid receptor subunit epsilon; IGF2, insulin-like growth factor 2; WDR82, WD repeat-containing protein 82; KRT19P2, keratin 19 pseudogene 2; FGFR3, fibroblast growth factor receptor 3; APC, adenomatous polyposis coli; CYP7A1, cytochrome P450 family 7 subfamily A member 1; NCAM1, neural cell adhesion molecule 1; IGF1R, insulin like growth factor 1 receptor.

and delta-like 1 (DLL1), and is regulated by 6 genes, including ZEB1, NR1H4 and MYC.

The TF Y in Yang 1 (YY1) was also obtained from a P-Match algorithm. YY1 is differentially expressed in HB (4), is targeted by hsa-miR-31 and hsa-miR-29a, and regulates CDK6.

Compared with the differentially expressed network, the associated network reveals more associations and provides a wider perspective to aid the understanding of the pathogenesis of HB.

Global network of $H B$. The topological associations derived from the D1, D2 and D3 datasets comprise the global network, which includes the associated and differentially expressed networks. The global network provides a more comprehensive perspective to study the pathogenesis of HB.

Comparison and analysis of the features of differentially expressed genes in the $H B$ networks. The present study analyzed the differentially expressed genes and associated factors obtained from the aforementioned miRNAs and genes pathways. The genes were classified according to the regulatory associations of their adjacent nodes with miRNAs derived from the three networks at various levels, in order to analyze the interacting feature of each gene.

A total of 30 differentially expressed genes, including CCND1, CTNNB1 and LIN28B, were analyzed in the present study. These genes may be classified into 7 classes, according to their adjacent nodes. The first class of genes consists of genes that possess 6 types of adjacent nodes. The adjacent nodes include 3 successors (downstream elements) and 3 predecessors (upstream elements). These genes include CTNNB1, EGFR, MYC, CCND1, MYCN, LIN28B and TP53. The following section focuses on MYC.

Table I shows the differentially expressed and regulatory associations of MYC with miRNAs. In total, 5 differentially expressed miRNAs, including hsa-miR-21, hsa-miR-17-5p, hsa-let-7a, hsa-let-7g and hsa-miR-371, target MYC; 13 miRNAs in the global network target MYC; and MYC regulates 
Table I. Regulatory associations between MYC and miRNAs.

\begin{tabular}{|c|c|c|}
\hline Network & miRNAs that target MYC & miRNAs that are regulated by MYC \\
\hline $\begin{array}{l}\text { Differentially } \\
\text { expressed }\end{array}$ & miR-17-5p, let-7a, let-7g, miR-21 and miR-371 & miR-18a, miR-17-5p, miR-221, let-7a and miR-195 \\
\hline Associated & miR-17-5p, let-7a, miR-21 and miR-34a & $\begin{array}{l}\text { miR-18a, miR-17-5p, miR-221, let-7a, miR-195, } \\
\text { miR-29b and miR-34a }\end{array}$ \\
\hline Global & $\begin{array}{l}\operatorname{miR}-17-5 p \text {, let-7a, miR-21, miR-34a, let-7g, } \\
\text { miR-145, miR-20a, miR-24, miR-26a, miR-34b, } \\
\text { miR-34c-5p, miR-378 and miR-98 }\end{array}$ & $\begin{array}{l}\text { miR-18a, miR-17-5p, miR-221, let-7a, miR-195, } \\
\text { miR-29b, miR-34a, let-7b, let-7c, let-7d, let-7e, } \\
\text { let-7f, let-7g, let-7i, miR-106a, miR-106b, miR-141, } \\
\text { miR-15a, miR-16-1, miR-18a, miR-19a, miR-19b, } \\
\text { miR-20b, miR-20b, miR-22, miR-23a, miR-23b, } \\
\text { miR-29a, miR-29b, miR-9, miR-92a and miR-93 }\end{array}$ \\
\hline
\end{tabular}

MYC, v-myc avian myelocytomatosis viral oncogene homolog; miRNA, microRNA; miR, microRNA; let, lethal.

Table II. Regulatory associations between hsa-miR-17-5p and various genes.

\begin{tabular}{|c|c|c|}
\hline Network & Genes that regulate hsa-miR-17-5p & Target genes of hsa-miR-17-5p \\
\hline $\begin{array}{l}\text { ifferentially } \\
\text { xpressed }\end{array}$ & MYCN, CCND1 and MYC & CCND1, MYC and CDKN1A \\
\hline Associated & MYCN, CCND1, MYC and NFKB1 & CCND1, MYC and CDKN1A \\
\hline lobal & $\begin{array}{l}\text { MYCN, CCND1, MYC, E2F1, ESR1, } \\
\text { NFKB1, SPI1, STAT5, TLX1, TLX3 and TNF }\end{array}$ & $\begin{array}{l}\text { CCND1, MYC, CDKN1A, APP, CCL1, BCL2L11, } \\
\text { BMPR2, CCND2, FBXO31, GPR137B, DNAJC27, } \\
\text { E2F3, ICAM1, E2F1, JAK1, MAP3K12 and BCL2 }\end{array}$ \\
\hline \multicolumn{3}{|c|}{$\begin{array}{l}\text { Hsa, Homo sapiens; miR, microRNA; MYCN, v-myc avian myelocytomatosis viral oncogene neuroblastoma derived homolog; CCN, cyclin; } \\
\text { MYC, v-myc avian myelocytomatosis viral oncogene homolog; CDKN1A, cyclin-dependent kinase inhibitor 1A; NFKB1, nuclear factor } \\
\text { of kappa light polypeptide gene enhancer in B-cells 1; E2F, E2F transcription factor; ESR1, estrogen receptor 1; APP, amyloid beta (A4) } \\
\text { precursor protein; CCL1, chemokine (C-C motif) ligand 1; BCL2, B-cell lymphoma 2; BCL2L11, BCL2-like 11; SPI1, Spi-1 proto-oncogene; } \\
\text { STAT5, signal transducer and activator of transcription 5A; TLX, T-cell leukemia homeobox; TNF, tumor necrosis factor; BMPR2, bone } \\
\text { morphogenetic protein receptor type II; FBXO31, F-box protein 31; GPR137B, G protein-coupled receptor 137B; DNAJC27, DnaJ (Hsp40) } \\
\text { homolog, subfamily C, member 27; ICAM1, intercellular adhesion molecule 1; JAK1, Janus kinase 1; MAP3K12, mitogen-activated protein } \\
\text { kinase kinase kinase 12. }\end{array}$} \\
\hline
\end{tabular}

Table III. Regulatory associations between NFKB1 and miRNAs.

\begin{tabular}{|c|c|c|}
\hline Network & miRNAs that target NFKB1 & miRNAs that are regulated by NFKB1 \\
\hline $\begin{array}{l}\text { Differentially } \\
\text { expressed }\end{array}$ & let-7a & miR-17-5p, miR-214, miR-224, miR-125b and miR-21 \\
\hline Associated & let- $7 \mathrm{a}$ & miR-17-5p, miR-214, miR-224, miR-125b, miR-21, miR-34a and miR-29b \\
\hline Global & $\begin{array}{l}\text { let-7a, miR-146a, miR-146b-1, } \\
\text { miR-15a and miR-9 }\end{array}$ & $\begin{array}{l}\text { miR-17-5p, miR-214, miR-224, miR-125b, miR-21, miR-34a, miR-29b, } \\
\text { miR-199a-5p, let-7a-3, let-7b, miR-10b, miR-125b-2, miR-146a, miR-155, } \\
\text { miR-16, miR-9, miR-29a, miR-365 and miR-448 }\end{array}$ \\
\hline
\end{tabular}

NFKB1, nuclear factor of kappa light polypeptide gene enhancer in B-cells 1; miRNA, microRNA; let, lethal; miR, microRNA.

5 miRNAs in the differentially expressed network, including hsa-miR-18a, hsa-miR-17-5p, hsa-miR-221, hsa-let-7a-2 and hsa-miR-195. A total of 4 and 13 miRNAs target MYC in the associated and global networks, respectively; and 7 and 32 miRNAs are regulated by MYC in the associated and global networks, respectively. According to these results, it is evident that miRNAs are able to affect directly the expression of multiple miRNAs. In addition, hsa-let-7a was observed to target MYC, and MYC regulated hsa-let-7a at all three network levels. Therefore, hsa-let-7a and MYC form a feedback loop, and are vital in the process of HB development. Mutations in MYC may directly influence other genes via miRNAs. For example, 
MYC influences the expression of TP53 by hsa-miR-221. Furthermore, the differential expression of miRNAs affects other miRNAs through MYC. For example, hsa-miR-17-5p targets MYC, and MYC regulates hsa-miR-195. Certain additional miRNAs in the global network are not involved in $\mathrm{HB}$, but aid the understanding of the pathogenesis of HB.

The second class of genes consists of genes that possess 4 types of adjacent nodes. MYCN regulates 3 miRNAs in the differentially expressed network, namely hsa-miR-18a, hsa-miR-17-5p and hsa-miR-221. However, hsa-miR-18a does not target any gene, indicating that hsa-miR-18a may directly act on $\mathrm{HB}$.

The third class of genes consists of genes that possess 3 types of adjacent nodes. This class includes IGF2, YY1, cyclin-dependent kinase inhibitor 1A (CDKN1A), adenomatous polyposis coli, cytochrome P450 family 7 subfamily A member 1 and fibroblast growth factor receptor 3 (FGFR3), which do not regulate any miRNAs in the three network levels. Therefore, these genes may be last actors in HB.

Neurogenic locus notch homolog protein 2 possesses 2 types of adjacent nodes, thus constituting the fourth class of genes, and is not suspected to be important in the pathogenesis of HB.

Comparison and analysis of the features of differentially expressed miRNAs in the HB networks. The same method was used to compare the differentially expressed miRNAs and genes. From the extracted data, 34 differentially expressed miRNAs, including hsa-miR-17-5p, hsa-miR-18a and hsa-miR-125a, were identified. To facilitate the understanding of the pathogenesis of $\mathrm{HB}$, the following section focuses on the miRNAs that are associated with other genes and, extracts the information regarding these associations.

The aforementioned 34 miRNAs were classified into 5 classes: The first class of miRNAs consists of miRNAs that possess 6 types of adjacent nodes. A total of 7 miRNAs, including hsa-miR-21, hsa-let-7a, hsa-let-7g, hsa-miR-125b, hsa-miR-221, hsa-miR-371 and hsa-miR-17-5p, were observed to have 3 successors and 3 predecessors. To use hsa-miR-17-5p as an example, Table II shows that 3 differentially expressed genes, namely CCND1, MYC and MYCN, regulate hsa-miR-17-5p; and 3 differentially expressed genes, namely CCND1, MYC and CDKN1A, are targeted by hsa-miR-17-5p. Therefore, CCND1, MYC and MYCN directly influence CCND1, MYC and CDKN1A through hsa-miR-17-5p. In total, 4 and 11 genes regulate hsa-miR-17-5p in the associated and global networks, respectively; and hsa-miR-17-5p targets 3 and 17 genes in the associated and global networks, respectively. In addition, certain miRNAs are affected by hsa-miR-17-5p through certain genes. For example, hsa-miR-17-5p targets MYC, which regulates miRNAs, including hsa-miR-18a and hsa-miR-195.

The second and third classes of miRNAs consist of miRNAs that possess 4 types of adjacent nodes. The miRNAs in the second class, specifically, have 1 predecessor and 3 successors. For example, hsa-miR-125a has no regulatory gene in the differentially expressed network or the associated network, but 2 genes share a regulatory pathway with hsa-miR-125a in the global network. In addition, TP53 and CDKN1A target hsa-miR-125a in the differentially expressed network, while a total of 3 and 19 genes target hsa-miR-18a in the associated and global networks, respectively.
In contrast, the third class of miRNAs has 3 predecessors and 1 successor. For example, 2 genes, namely MYC and MYCN, regulate hsa-miR-18a in the differentially expressed network, and 2 and 9 genes regulate hsa-miR-18a in the associated and global networks, respectively. However in the global network, only 2 genes target hsa-miR-18a.

The fourth class consists of miRNAs that only possess 3 successors, such as hsa-miR-100, which is targeted by FGFR3 in the differentially expressed and associated networks, while 6 genes target hsa-miR-100 in the global network. The fifth class has $<3$ adjacent nodes. For example, hsa-miR-148a is only regulated by 3 genes, and targets 7 genes in the global network.

Comparison and analysis of the features of predicted TFs in the HB networks. The analysis of predicted TFs was conducted following the aforementioned method. A total of $5 \mathrm{TFs}$, namely ZEB1, YY1, REL, RELA and NFKB1, regulate miRNAs in the associated network. The first class of TFs consists of TFs that possess 6 types of adjacent nodes, specifically 3 successors and 3 predecessors. NFKB1, an example of a TF from the first class, possesses 6 types of adjacent nodes. Table III shows that the differentially expressed miRNA hsa-let-7a targets NFKB1, which regulates the differentially expressed miRNAs hsa-miR-214, hsa-miR-224, hsa-miR-125b and hsa-miR-17-5p. In the associated and global networks, respectively, 1 and 5 miRNAs target NFKB1, which in turn regulates 7 and 19 miRNAs in the above networks. In the present study, NFKB1 was not observed to be a differentially expressed TF, but exhibited a close association with differentially expressed miRNAs. Since NFKB1 is only targeted by hsa-let-7a-2 in the associated network, it may be possible to conclude that hsa-let-7a-2 directly results in expression errors involving other miRNAs through NFKB1. The miRNAs that are not differentially expressed possess certain associations with HB.

The second class of TFs, which includes RELA, REL, ZEB1 and YY1, consists of TFs that possess 4 types of adjacent nodes. RELA regulates the differentially expressed miRNA hsa-miR-21. However, none of the differentially expressed miRNAs target RELA, while hsa-miR-373 targets RELA in the associated network. As for YY1, none of the differentially expressed miRNAs regulate or target YY1. In the associated network, hsa-miR-34a targets YY1, which regulates hsa-miR-29b. ZEB1 and REL are similar, in that no miRNAs target them. Therefore, RELA may be the first actor in the pathogenesis of HB. Future studies are recommended to account for these important factors.

\section{Discussion}

In the present study, certain data were collected from authoritative websites and pertinent literature, while certain data were obtained using P-Match. A total of three networks at various levels, including differentially expressed, associated and global networks, were constructed in order to analyze all the experimentally validated data. The pathways involving TFs, target genes, miRNAs and host genes were analyzed. Since these genes and miRNAs are not isolated, their development or mutation may affect other genes or miRNAs that are associated with HB.

In the present study, numerous important genes and miRNAs were identified. In total, 3 differentially expressed 
TFs, namely CCND1, MYC and EGFR, were observed to form FFLs that correspond to hsa-miR-17-5p, hsa-let-7a and hsa-miR-21, respectively. MYCN regulates 3 miRNAs (hsa-miR-17-5p, hsa-miR-18a and hsa-miR-221) in the differentially expressed and associated networks, and notably, no miRNAs target MYCN. The associated miRNA hsa-miR-34a targets 13 genes, and is regulated by 6 TFs. Following the P-Match method, 6 TFs were obtained, which may be also important, as they suggest potential associations between miRNAs and TFs. The associations between miRNAs and TFs are complex, and lead to the interaction between miRNAs and TFs and the regulation of $\mathrm{HB}$.

Core genes and miRNAs are unlikely to only affect one type of cancer, and may play a significant role in other types of cancer. A previous study indicated that $\mathrm{MYCN}$ is not only differentially expressed in HB, but also in Wilms' tumor (33). Furthermore, hsa-miR-373 is overexpressed in HB, and is also differentially expressed in retinoblastoma (34). Future studies should focus on the genes and miRNAs that are common in various types of cancer. The authoritative data and pathways discussed in the present study provide a novel perspective for the understanding of $\mathrm{HB}$.

\section{Acknowledgements}

The present study was supported by the National Natural Science Foundation of China (Beijing, China; grant no. 60905022).

\section{References}

1. De Ioris M, Brugieres L, Zimmermann A, Keeling J, Brock P, Maibach R, Pritchard J, Shafford L, Zsiros J, Czaudzerna P and Perilongo G: Hepatoblastoma with a low serum alpha-fetoprotein level at diagnosis: The SIOPEL group experience. Eur J Cancer 44: 545-550, 2008.

2. Lack EE, Neave $C$ and Vawter GF: Hepatoblastoma. A clinical and pathologic study of 54 cases. Am J Surg Pathol 6: 693-705, 1982 .

3. Herzog CE, Andrassy RJ and Eftekhari F: Childhood cancers: Hepatoblastoma. Oncologist 5: 445-453, 2000.

4. Shin E, Lee KB, Park SY, Kim SH, Ryu HS, Park YN, Yu E and Jang JJ: Gene expression profiling of human hepatoblastoma using archived formalin-fixed and paraffin-embedded tissues. Virchows Arch 458: 453-465, 2011.

5. Baynam GS and Goldblatt J: A child with an FGFR3 mutation, a laterality disorder and an hepatoblastoma: Novel associations and possible gene-environment interactions. Twin Res Hum Genet 13: 297-300, 2010.

6. Zimmermann A: The emerging family of hepatoblastoma tumours: From ontogenesis to oncogenesis. Eur J Cancer 41: 1503-1514, 2005.

7. Hobert O: Gene regulation by transcription factors and microRNAs. Science 319: 1785-1786, 2008.

8. Latchman DS: Transcription factors: An overview. Int J Biochem Cell Biol 29: 1305-1312, 1997.

9. Bartel DP: MicroRNAs: Target recognition and regulatory functions. Cell 136: 215-233, 2009.

10. Magrelli A, Azzalin G, Salvatore M, Viganotti M, Tosto F, Colombo T, Devito R, Di Masi A, Antoccia A, Lorenzetti S, et al: Altered microRNA expression patterns in hepatoblastoma patients. Transl Oncol 2: 157-163, 2009.

11. Hobert O: Common logic of transcription factor and microRNA action. Trends Biochem Sci 29: 462-468, 2004

12. Wang J, Lu M, Qiu C and Cui Q: TransmiR: A transcription factor-microRNA regulation database. Nucleic Acids Res 38: D119-D122, 2010.
13. Griffiths-Jones S, Grocock RJ, van Dongen S, Bateman A and Enright AJ: miRBase: MicroRNA sequences, targets and gene nomenclature. Nucleic Acids Res 34: D140-D144, 2006.

14. Naeem H, Küffner R and Zimmer R: MIRTFnet: Analysis of miRNA regulated transcription factors. PLoS One 6: e22519, 2011.

15. Volinia S, Calin GA, Liu CG, Ambs S, Cimmino A, Petrocca F, Visone R, Iorio M, Roldo C, Ferracin M, et al: A microRNA expression signature of human solid tumors defines cancer gene targets. Proc Natl Acad Sci USA 103: 2257-2261, 2006.

16. Ishimoto K, Nakamura H, Tachibana K, Yamasaki D, Ota A, Hirano K, Tanaka T, Hamakubo T, Sakai J, Kodama T and Doi T: Sterol-mediated regulation of human lipin 1 gene expression in hepatoblastoma cells. J Biol Chem 284: 22195-22205, 2009.

17. Brand AH and Perrimon N: Targeted gene expression as a means of altering cell fates and generating dominant phenotypes. Development 118: 401-415, 1993.

18. Rodriguez A, Griffiths-Jones S, Ashurst JL and Bradley A: Identification of mammalian microRNA host genes and transcription units. Genome Res 14: 1902-1910, 2004.

19. Gennarino VA, Sardiello M, Avellino R, Meola N, Maselli V, Anand S, Cutillo L, Ballabio A and Banfi S: MicroRNA target prediction by expression analysis of host genes. Genome Res 19: 481-490, 2009

20. Papadopoulos GL, Reczko M, Simossis VA, Sethupathy P and Hatzigeorgiou AG: The database of experimentally supported targets: A functional update of TarBase. Nucleic Acids Res 37: D155-D158, 2009.

21. Kozomara A and Griffiths-Jones S: miRBase: Integrating microRNA annotation and deep-sequencing data. Nucleic Acids Res 39: D152-D157, 2011.

22. Garfield E: "Science Citation Index" - A new dimension in indexing. Science 144: 649-654, 1964.

23. Chekmenev DS, Haid C and Kel AE: P-Match: Transcription factor binding site search by combining patterns and weight matrices. Nucleic Acids Res 33 (Web Server): W432-W437, 2005.

24. Fujita PA, Rhead B, Zweig AS, Hinrichs AS, Karolchik D, Cline MS, Goldman M, Barber GP, Clawson H, Coelho A, et al: The UCSC Genome Browser database: Update 2011. Nucleic Acids Res 39: D876-D882, 2011.

25. Bao J, Li D, Wang L, Wu J, Hu Y, Wang Z, Chen Y, Cao X, Jiang C, Yan W and Xu C: MicroRNA-449 and microRNA-34b/c function redundantly in murine testes by targeting E2F transcription factor-retinoblastoma protein (E2F-pRb) pathway. J Biol Chem 287: 21686-21698, 2012.

26. Jiang Q, Wang Y, Hao Y, Juan L, Teng M, Zhang X, Li M, Wang $\mathrm{G}$ and Liu Y: miR2Disease: A manually curated database for microRNA deregulation in human disease. Nucleic Acids Res 37: D98-104, 2009.

27. Golan D, Levy C, Friedman B and Shomron N: Biased hosting of intronic microRNA genes. Bioinformatics 26: 992-995, 2010.

28. Cairo S, Wang Y, de Reyniès A, Duroure K, Dahan J, Redon MJ, Fabre M, McClelland M, Wang XW, Croce CM and Buendia MA: Stem cell-like micro-RNA signature driven by Myc in aggressive liver cancer. Proc Natl Acad Sci USA 107: 20471-20476, 2010.

29. López-Terrada D, Gunaratne PH, Adesina AM, Pulliam J, Hoang DM, Nguyen Y, Mistretta TA, Margolin J and Finegold MJ: Histologic subtypes of hepatoblastoma are characterized by differential canonical Wnt and Notch pathway activation in $\mathrm{DLK}^{+}$precursors. Hum Pathol 40: 783-794, 2009.

30. King CE, Wang L, Winograd R, Madison BB, Mongroo PS, Johnstone CN and Rustgi AK: LIN28B fosters colon cancer migration, invasion and transformation through let-7-dependent and -independent mechanisms. Oncogene 30: 4185-4193, 2011.

31. Viswanathan SR and Daley GQ: Lin28: A microRNA regulator with a macro role. Cell 140: 445-449, 2010.

32. Giardiello FM, Petersen GM, Brensinger JD, Luce MC, Cayouette MC, Bacon J, Booker SV and Hamilton SR: Hepatoblastoma and APC gene mutation in familial adenomatous polyposis. Gut 39: 867-9, 1996.

33. Zirn B, Hartmann O, Samans B, Krause M, Wittmann S, Mertens F, Graf N, Eilers M and Gessler M: Expression profiling of Wilms tumors reveals new candidate genes for different clinical parameters. Int J Cancer 118: 1954-1962, 2006.

34. Li J, Xu ZW, Wang KH, Wang N, Li DQ and Wang S: Networks of microRNAs and genes in retinoblastomas. Asian Pac J Cancer Prev 14: 6631-6636, 2014. 